\title{
Evaluación de la geometría endonasal en población chilena sana mediante rinometría acústica
}

\section{Acoustic rhinometry nasal geometry assessment in Chilean healthy population}

\author{
Alejandro 0jeda S1, Daniel Muñoz S², Héctor Bahamonde \$1. $^{1}$.
}

\begin{abstract}
RESUMEN
Introducción: La rinometría acústica es una técnica de laboratorio que permite objetivar la geometría de la cavidad nasal. En nuestro medio aún hay discusión acerca de su utilidad y no existen estudios referenciales.

Objetivos: Establecer parámetros referenciales para rinometría acústica en población chilena adulta sana.

Material y método: Se seleccionaron prospectivamente adultos voluntarios sanos para evaluación rinológica y antropométrica. Se midieron las áreas mínimas de sección transversal y volúmenes endonasales en estado basal y posterior a la administración tópica de vasocontrictor.

Resultados: Se obtuvieron 83 individuos adultos sanos, de ellos el 56,63\% son hombres, con edad promedio de 24,3 años. Los parámetros rinométricos fueron: MCA1 (área

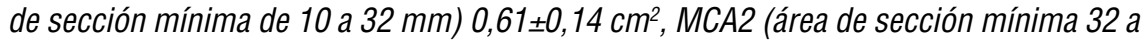
$64 \mathrm{~mm}$ ) $0.62 \pm 0.018 \mathrm{~cm}^{2}$, Vol1 (volumen entre narina y $32 \mathrm{~mm}$ ) $1.99 \pm 0.039 \mathrm{~cm}^{3}$, Vol2 (volumen entre $32 \mathrm{~mm}$ y $64 \mathrm{~mm}$ ) 4,43 $\pm 0,15 \mathrm{~cm}^{3}$. Posvasoconstrictor se encontró aumento significativo en áreas y volúmenes salvo en Vol1.

Discusión: Es fundamental definir rangos normales de los diámetros de la cavidad nasal, para aplicar dicha información en la evaluación de la patología rinosinusal que supone anormalidad de la geometría endonasal.

Conclusión: Los valores encontrados mostraron diferencias significativas en los parámetros rinométricos por sexo y respuesta posvasoconstricción, sin diferencias en relación a la antropometría.
\end{abstract}

Palabras clave: Rinometría acústica, nasal.

\section{ABSTRACT}

Introduction: Acoustic rhinometry is a laboratory technique that allows assess nasal airway. In our area there is still debate regarding its usefulness and there are no local studies for reference values.

Aim: To establish acoustic rhinometry reference values for healthy adult Chilean population.

Material and method: Prospective clinical and analytical study. Healthy adult volunteers were selected for rhinological and anthropometric evaluation (body surface

Médico. Servicio de Otorrinolaringología, Hospital Clínico Universidad de Chile.

2 Médico Cirujano. Universidad de Chile. 
area). Minimum areas of cross section and endonasal volumes in each nostril at baseline and after topical administration of a vasoconstrictor were measured.

Results: 83 healthy adult subjects were obtained, $56.63 \%$ males and $43.37 \%$ female, mean age of 24.3 years. Acoustic rhinometry parameters were: $0.61 \pm 0.14 \mathrm{~cm} 2 \mathrm{MCA1}$, MCA2 $0.62 \pm 0.018 \mathrm{~cm} \mathrm{2,} 1.99 \pm 0.039 \mathrm{~cm} 3$ Vol1, Vol $24.43 \pm 0.15 \mathrm{~cm} 3$. Post vasoconstrictor significant increase except in areas and volumes Vol1 was found.

Discussion: It is essential to define the normal range of internal diameters of the nasal cavity, and then apply this information in evaluating endonasal geometry abnormality.

Conclusion: Significant differences in acoustic rhinometry parameters by sex and postvasoconstriction response were found, without differences in relation to anthropometry.

Key words: Acustic rhinometry, nose.

\section{INTRODUCCIÓN}

La obstrucción nasal es un síntoma muy frecuente en la práctica clínica otorrinolaringológica, sin embargo, la objetivación de este síntoma en la especialidad sigue siendo una fuente de discrepancia, esto fundamentalmente debido a que la correlación entre los hallazgos de la exploración clínica y el laboratorio, a menudo entregan resultados dispares con la valoración subjetiva que nos entrega el paciente sobre este síntoma ${ }^{1}$.

La rinometría acústica (RA) aparece como una técnica de laboratorio que permite objetivar la vía aérea nasal y corresponde a un examen no invasivo que permite la medición objetiva de la geometría endonasal mediante el estudio de reflexión de señales acústicas². Mediante un análisis computacional es posible calcular áreas mínimas de sección transversal (MCA) en centímetros cuadrados y volúmenes endonasales (VE) en centímetros cúbicos. Las MCA se localizan usualmente a nivel de la válvula nasal y del cornete medio y los volúmenes endonasales entre estos segmentos ${ }^{3}$.

La RA es uno de los exámenes de mayor uso en la evaluación de la patología nasosinusal, demostrando en múltiples publicaciones su utilidad en el estudio de obstrucción nasal, del ciclo nasal, hipertrofia adenoidea, roncopatía, malformaciones coanales congénitas y en la evaluación de la geometría endonasal previo a cirugía nasal ${ }^{4,5}$.

Sus valores varían por múltiples factores, como: edad, sexo, antropometría, técnica empleada y la raza, siendo este último considerado por múltiples estudios como el principal factor ${ }^{6,7}$.

A pesar del extendido uso a nivel mundial y de las publicaciones sobre esta técnica en población normal y patológica, existe aún discusión en rela- ción a su real utilidad y debate en relación a los valores de referencia, pues nuestro medio carece de estudios sobre valores referenciales.

\section{OBJETIVO}

Nuestro objetivo es establecer valores de referencia para RA en población chilena sana. Además, se correlacionará los hallazgos con variables demográficas como el sexo y variables antropométricas como el área de superficie corporal (ASC). Se analizará el efecto de un vasoconstrictor tópico $(\mathrm{Vc})$ en los valores obtenidos y finalmente compararemos nuestros resultados con otras series que hallan sido obtenidas con orientación étnica.

\section{MATERIAL Y MÉTODO}

Estudio clínico prospectivo y analítico. Sobre un universo de 126 participantes se seleccionaron 83 adultos voluntarios sanos para evaluación rinológica en el Servicio de Otorrinolaringología del Hospital Clínico de la Universidad de Chile desde mayo de 2010 hasta noviembre del año 2011, previa firma de un consentimiento informado validado por el Comité de Ética de nuestro centro.

\section{Selección de sujetos}

La elección de los participantes que se incluyeron dentro del estudio se rigió según protocolo diseñado por los autores del trabajo para cumplir los objetivos propuestos. Se incluyeron:

- Pacientes voluntarios sanos de nacionalidad chilena que estén dispuestos a realizarse una 
rinometria acústica, llenar formulario adjunto y firmar un consentimiento informado.

- Edad de 21 a 50 años.

- Pacientes sin historia de obstrucción nasal permanente 0 aguda actual.

Asimismo, se excluyeron del estudio:

- Pacientes con antecedentes de enfermedad crónica sistémica y/o localizada tales como: rinitis alérgica, rinosinusitis, poliposis nasosinusal.

- Pacientes con antecedente de cirugía rinosinusal y/o trauma nasal.

- Pacientes que estén utilizando alguna droga descongestionante, antihistamínica, vasoconstrictora o corticoterapia inhalatoria.

- Pacientes con hallazgos patológicos acentuados en el examen otorrinolaringológico como: hipertrofia de cornetes, septodesviación nasal 0 infección rinosinusal.
A este grupo se le aplicó el protocolo de evaluación de RA (Figura 1), siendo éste el instrumento de recolección de la información, el cual incluye la historia clínica, evaluación antropométrica con obtención de ASC (fórmula de Dubois \& Dubois) medición elegida por ser una mejor representación de la dimensión corporal por sobre el índice de masa corporal ${ }^{2}$ y el examen rinoscópico.

\section{Medición con rinometría acústica}

La medición se efectuó con un equipo Rhinoscan ${ }^{\circledR}$ (SRE 2000 A/S Denmark) siguiendo las recomendaciones del comité de estandarización de evaluación de vía aérea4:

- Previo al test los sujetos son sometidos a higiene nasal con atomización endonasal de solución fisiológica isotónica al 0,9\% más rinoscopía anterior.

$\begin{array}{lll}\text { Respirador bucal } & \bigcirc \text { sí } & \bigcirc \text { no } \\ \text { Trauma nasal severo } & \bigcirc \text { sí } & \bigcirc \text { no } \\ \text { Obstrucción nasal aguda o permanente } & \bigcirc \text { sí } & \bigcirc \text { no } \\ \text { Enfermedad sistémica } & \bigcirc \text { sí } & \bigcirc \text { no ¿Cuál? } \\ \text { Enfermedad atópica } & \bigcirc \text { sí } & \bigcirc \text { no ¿Cuál? } \\ \text { Sinusitis recurrente } & \bigcirc \text { sí } & \bigcirc \text { no } \\ \text { Poliposis nasosinusal } & \bigcirc \text { sí } & \bigcirc \text { no } \\ \text { Cirugía rinsinusal } & \bigcirc \text { sí } & \bigcirc \text { no } \\ \text { Uso de alguna droga descongestionante } & \bigcirc \text { sí } & \bigcirc \text { no }\end{array}$

Otros

\section{Examen físico:}

$\begin{array}{ll}\text { Septodesviación nasal } & \bigcirc \text { no } \bigcirc \text { leve } \bigcirc \text { mod-sev } \\ \text { Hipertrofia de cornetes } & \bigcirc \text { no } \bigcirc \text { leve } \bigcirc \text { mod-sev } \\ \text { Espejo de Glatzel } & \bigcirc \text { normal } \bigcirc \text { anormal } \\ \text { Poliposis nasal } & \bigcirc \text { sí } \bigcirc \text { no } \\ \text { Signos inflamatorios-infecciosos endonasales } & \bigcirc \text { sí } \bigcirc \text { no } \\ \text { Perforación septal } & \bigcirc \text { sí } \bigcirc \text { no }\end{array}$

Rinometría acústica

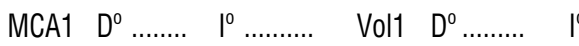

MCA2 $D^{0} \ldots \ldots . .1^{0} \ldots \ldots \ldots . . \quad$ Vol $2 D^{0} \ldots \ldots \ldots 1^{0}$

Posvasocnstrictor:

MCA1 $D^{0} \ldots \ldots . .\left.\right|^{0} \ldots \ldots \ldots . . \quad$ Voll $\left.D^{0} \ldots \ldots \ldots\right|^{\circ}$

MCA2 $D^{0} \ldots \ldots . . I^{0} \ldots \ldots \ldots . \quad$ Vol2 $D^{0} \ldots \ldots \ldots$

Examinadores

Figura 1. Protocolo de evaluación por rinometría acústica, Servicio de Otorrinolaringología, Hospital Clínico Universidad de Chile. 
- Luego permanecen sentados por 20 minutos en una habitación con temperatura constante, climatizada por aire acondicionado con los niveles de ruido ambiental requeridos para el examen.

- Se elige un adaptador adecuado para el tamaño del vestíbulo nasal para cada paciente, teniendo la precaución de no deformar el vestíbulo nasal al aplicar el adaptador.

- El tubo del rinómetro se posiciona paralelo al dorso nasal con una adecuada adaptación a la narina que se sella con gel neutro para evitar fuga de ondas sonoras.

- Los sujetos durante la medición permanecen quietos con la cabeza cómodamente apoyada, con la boca cerrada evitando tragar o mover la lengua.

- La curva analizada se obtiene en un periodo de pausa respiratoria luego de una exhalación nasal voluntaria.

- Se realizan 3 mediciones en cada fosa nasal registrando el promedio de estos valores para el análisis posterior.

- Se repite este mismo método luego de 10 minutos de la aplicación endonasal de un vasoconstrictor (Vc) tópico (oximetazolina 0,05\%).

Se determinaron en cada fosa nasal las MCA 1 (10 a $32 \mathrm{~mm}$ ), MCA 2 (32 a $64 \mathrm{~mm}$ ), Vol 1 (entre narina y $32 \mathrm{~mm}$ ) y Vol 2 (entre $32 \mathrm{~mm}$ y $64 \mathrm{~mm}$ ). Se realizaron estas mediciones en estado basal y posterior a la administración tópica endonasal de oximetazolina al $0,05 \%$ como Vc.

\section{Análisis estadístico}

El análisis de datos se efectuó con software STATA ${ }^{\circledR} 12$ (StataCorp. 2011. College Station, TX: StataCorp LP.), se utilizó media y desviación estándar para variables cuantitativas además de la prueba de t-student para comparación de medias y el coeficiente de correlación lineal de Pearson para cuantificar asociación entre variables. Se consideró como significativo un valor $p<0,05$.

\section{RESULTADOS}

Se obtuvo finalmente un total de 83 individuos adultos sanos, de los cuales el $56,63 \% \quad(n=47)$ correspondió a sexo masculino y el $43,37 \%(n=36)$ al sexo femenino. La edad promedio fue de $24,3 \pm 2,28$ años en un rango de 18 a 35 .

Para la población total los promedios encontrados se detallan en la Tabla 1, destacando menor área de sección endonasal en MCA1 con respecto a MCA2 y menor volumen endonasal en Vol1 en relación a Vol2. Además se encontraron diferencias estadísticamente significativas al comparar la geometría endonasal en estado basal versus posvasoconstricción en MCA1 y Vol 2 siendo mayores las áreas y volúmenes en la mediciones posterior a la instilación tópica de oximetazolina.

Al comparar valores basales obtenidos según sexo, encontramos diferencias estadísticamente significativas en los parámetros MCA1 y Vol1 con valores superiores en el caso de los hombres (Tabla 2).

Tabla 1. Parámetros rinométricos basales y posvasoconstricción

\begin{tabular}{|lccr|}
\hline & Basal & PosVc & P value \\
\hline MCA 1 $\left(\mathrm{cm}^{2}\right)$ & $0,61 \pm 0,14$ & $0,67 \pm 0,02$ & 0,0066 \\
MCA 2 $\left(\mathrm{cm}^{2}\right)$ & $0,62 \pm 0,018$ & $0,83 \pm 0,087$ & 0,0198 \\
Vol 1 $\left(\mathrm{cm}^{3}\right)$ & $1,99 \pm 0,039$ & $2,02 \pm 0,03$ & 0,1434 \\
Vol 2 $\left(\mathrm{cm}^{3}\right)$ & $4,43 \pm 0,15$ & $5,68 \pm 0,20$ & $<0,0001$ \\
\hline
\end{tabular}

Tabla 2. Comparación según sexo de resultados de parámetros rinométricos

\begin{tabular}{|lccr|}
\hline & Mujeres & Hombres & P Value \\
\hline MCA 1 $\left(\mathrm{cm}^{2}\right)$ & $0,57 \pm 0,19$ & $0,64 \pm 0,02$ & 0,0182 \\
MCA 2 $\left(\mathrm{cm}^{2}\right)$ & $0,60 \pm 0,02$ & $0,64 \pm 0,02$ & 0,3587 \\
Vol 1 $\left(\mathrm{cm}^{3}\right)$ & $1,76 \pm 0,05$ & $2,16 \pm 0,04$ & $<0,0001$ \\
Vol 2 $\left(\mathrm{cm}^{3}\right)$ & $4,14 \pm 0,20$ & $4,65 \pm 0,23$ & 0,1145 \\
\hline
\end{tabular}


Tabla 3. Comparación de resultados según sexo de parámetros rinométricos posvasoconstricción

\begin{tabular}{|lrrr|}
\hline & Mujeres & Hombres & P Value \\
\hline MCA 1 $\left(\mathrm{cm}^{2}\right)$ & $0,64 \pm 0,04$ & $0,68 \pm 0,02$ & 0,4161 \\
MCA 2 $\left(\mathrm{cm}^{2}\right)$ & $0,94 \pm 0,19$ & $0,74 \pm 0,02$ & 0,2536 \\
Vol 1 $\left(\mathrm{cm}^{3}\right)$ & $1,84 \pm 0,04$ & $2,15 \pm 0,04$ & $<0,0001$ \\
Vol 2 $\left(\mathrm{cm}^{3}\right)$ & $5,47 \pm 0,29$ & $5,83 \pm 0,27$ & 0,3789 \\
\hline
\end{tabular}

Al comparar valores posadministración de $\mathrm{Vc}$ obtenidos según sexo, encontramos una diferencia estadísticamente significativa sólo en el parámetro Vol1 con un valor superior en el caso de los hombres (Tabla 3).

Para cuantificar la asociación entre el ASC y las mediciones rinométricas se utilizó el coeficiente de correlación lineal de Pearson. Sólo se encontró una correlación de importancia entre el vol1 y el área de

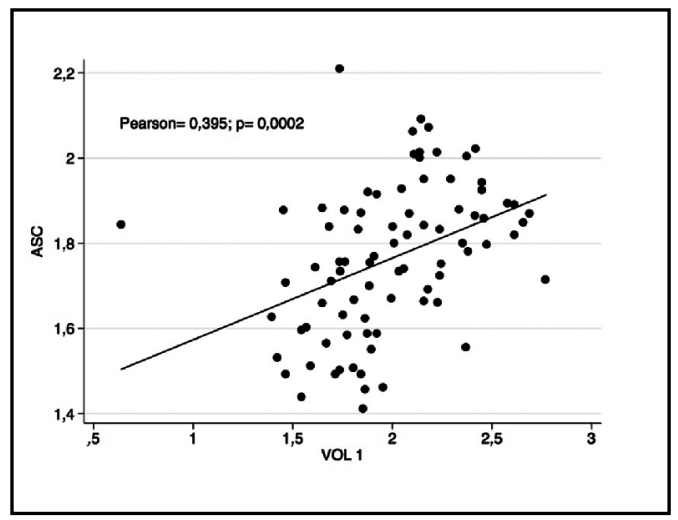

Figura 2. Correlación entre área de superficie corporal y volumen endonasal 1.

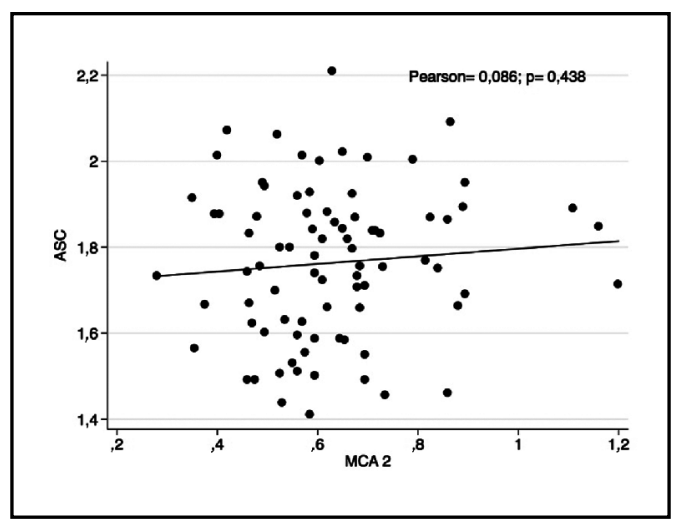

Figura 4. Correlación entre área de superficie corporal y MCA2. superficie corporal obteniendo un Pearson de 0,395 y un $p$-value de 0,0002 (Figura 2). No se encontraron coeficientes significativos al analizar el ASC con el resto de los parámetros rinométricos (Figuras 3, 4, 5), observando en las gráficas sólo patrones sugerentes de correlación en las nubes de dispersión. Al correlacionar MCA y volumen endonasal promedio entre el estado basal y posvasoconstrictor, se obtiene una correlación es-

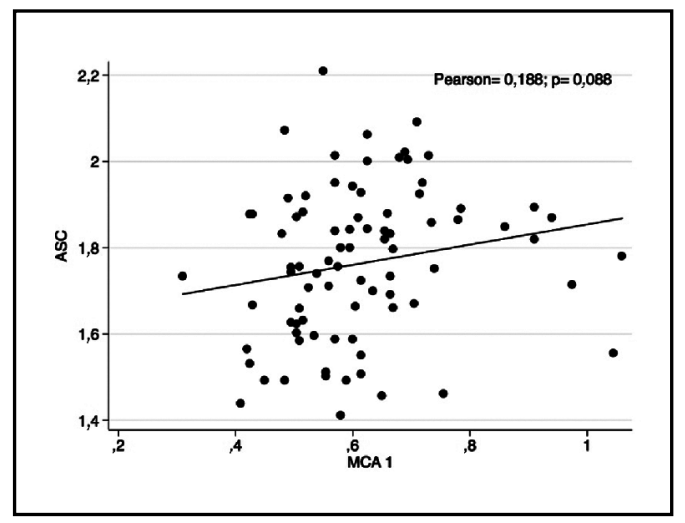

Figura 3. Correlación entre área de superficie corporal y MCA1.

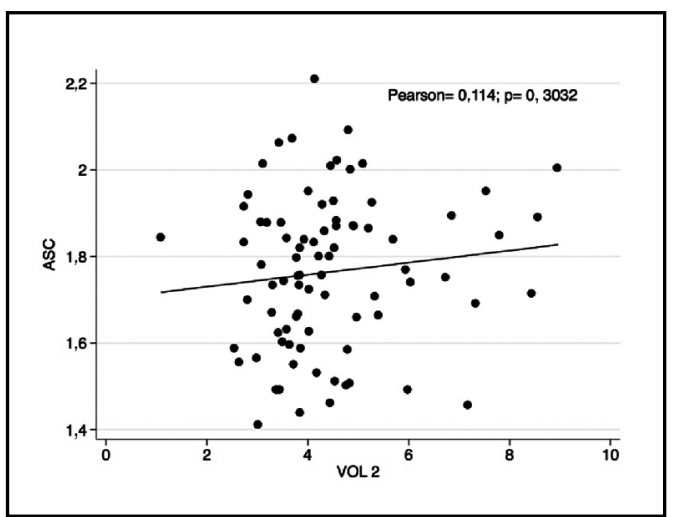

Figura 5. Correlación entre área de superficie corporal y Vol2. 


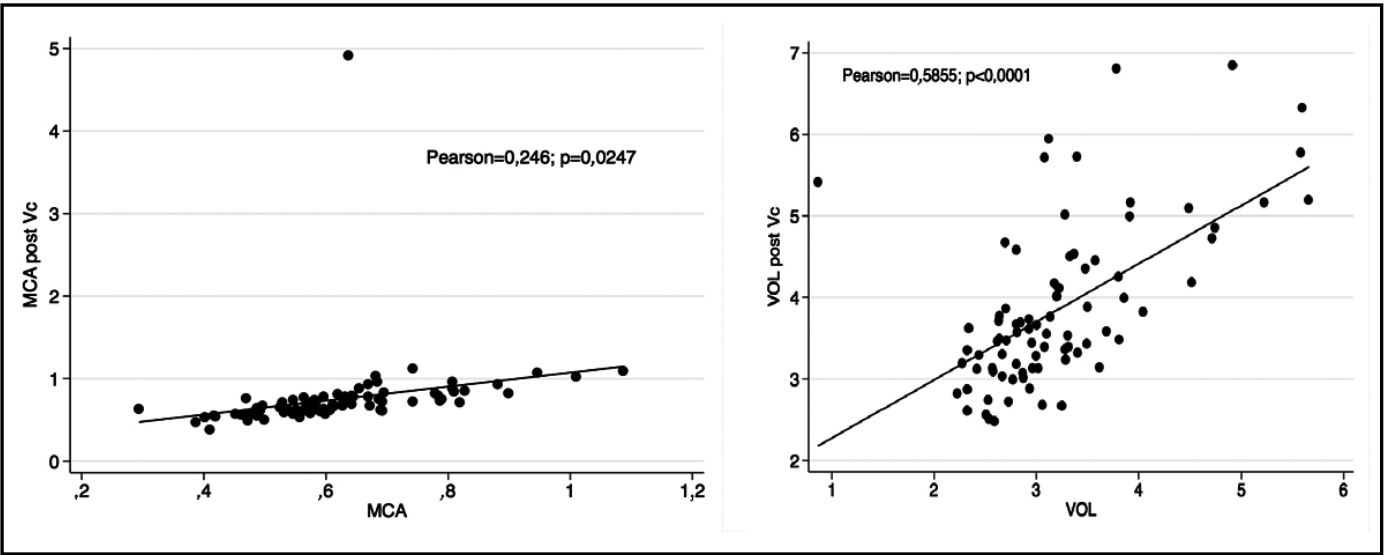

Figura 6. Correlacion entre MCA promedio y volumen promedio, pre y posvasoconstrictor.

tadísticamente significativa (Pearson $=0,246 ; p$ $=0,0247$ ) (Figura 6).

\section{DISCUSIÓN}

Durante los últimos años ha existido un gran interés en la objetivación de la obstrucción nasal, para esto se incluyen métodos de laboratorio como la rinomanometría y la RA. La rinomanometría entrega información sobre flujos aéreos y presiones intranasales mientras que la RA aporta información estática de la geometría endonasal. Ambos métodos entregan datos complementarios para hacer más objetiva esta valoración'1.

Desde el punto de vista clínico, es fundamental definir los rangos normales de los diámetros internos de la cavidad nasal, esto para luego utilizar dicha información en patología rinosinusal en donde debiéramos encontrar parámetros de anormalidad de la geometría endonasal.

Las investigaciones que comparan los valores de RA entre diferentes poblaciones se han transformado en una interesante área de estudio. En el estudio clásico de Corey y cols ${ }^{5}$, se encontró que sólo la MCA mostraba diferencias significativas al comparar poblaciones de origen africano, caucásicos y asiáticos, pero que no habían diferencias entre grupos caucásicos y asiáticos. En este mismo estudio otros parámetros rinométricos (volúmenes endonasales) no mostraron diferencias significativas. A pesar de los múltiples estudios que muestran diferencias entre grupos raciales, aún son insuficientes los datos como para determinar certeramente las variaciones de la geometría endonasal entre diferentes poblaciones.

En el análisis sobre los resultados obtenidos de los parámetros rinométricos de este estudio, se encontraron diferencias significativas entre valores basales y posVc, en todos a excepción del análisis en Vol1. No se encontró diferencias significativas en los valores encontrados al comparar sexo, salvo en el Vol1 y MCA1 basal y Vol1 posVc, con áreas y volúmenes superiores para el sexo masculino. No se encontró correlación entre los valores hallados y variables antropométricas, salvo en la correlación del ASC con Vol1, donde una mayor dimensión corporal se asoció a un mayor volumen endonasal anterior.

En relación al análisis étnico, los valores encontrados para las MCA y los volúmenes endonasales en nuestro estudio muestran similitudes interesantes con los datos de otras series ${ }^{5}$, por ejemplo, al comparar MCA en población asiática, caucásica y de nuestra serie, encontramos valores de 0,61 $\pm 0,6$, $0,61 \pm 0,3$ y $0,61 \pm 0,14$ respectivamente, evidenciando semejanzas evidentes. Una comparación extensa entre los distintos parámetros rinométricos según etnia publicados en la literatura se presentan en la Tabla 4.

\section{CONCLUSIONES}

La RA es una herramienta validada universalmente para la objetivación de la sintomatología obstructiva 
Tabla 4. Valores referenciales de RA (áreas y volúmenes nasales) en adultos sanos de diferentes razas, pre y posvasoconstrictor, reportados en la literatura

\begin{tabular}{|c|c|c|c|c|c|c|}
\hline \multirow[b]{2}{*}{ Autor (año) } & \multirow[b]{2}{*}{$\begin{array}{l}N^{0} \\
\text { pctes. }\end{array}$} & \multirow[b]{2}{*}{ País/etnia } & \multicolumn{2}{|c|}{ Prevasoconstrictor } & \multicolumn{2}{|c|}{ Posvasoconstrictor } \\
\hline & & & $\begin{array}{l}\text { MCA } \\
\text { (prom. } \pm D E \text { ) }\end{array}$ & $\begin{array}{l}\text { VE } \\
\text { (prom. } \pm D E \text { ) }\end{array}$ & $\begin{array}{l}\text { MCA } \\
\text { (prom. } \pm D E \text { ) }\end{array}$ & $\begin{array}{l}\text { VE } \\
\text { (prom. } \pm D E \text { ) }\end{array}$ \\
\hline $\operatorname{Miman}^{7}(2006)$ & 124 & Turquía & $0,48 \pm 0,21$ & $1,56 \pm 0,78$ & $0,51 \pm 0,15$ & $2,14 \pm 0,19$ \\
\hline Cakmak $^{8}$ (2005) & 25 & Turquía & $0,61 \pm 0,12$ & $1,42 \pm 0,51$ & - & - \\
\hline Samolinski9 (2007) & 324 & Polonia & $0,51 \pm 0,24$ & - & - & - \\
\hline Mohebbi ${ }^{10}(2008)$ & 180 & Irán & $0,45 \pm 0,17$ & -- & $0,52 \pm 0,18$ & - \\
\hline Tantilipikorn ${ }^{11}$ (2008) & 135 & Tailandia & $0,61 \pm 0,60$ & $3,66 \pm 0,67$ & $0,64 \pm 0,14$ & $4,18 \pm 0,75$ \\
\hline Straszek $^{12}(2007)$ & 146 & Dinamarca & $0,61 \pm 0,30$ & $3,73 \pm, 70$ & $0,73 \pm 0,40$ & $5,28 \pm 0,21$ \\
\hline \multirow[t]{3}{*}{ Corey $^{5}(1998)$} & 24 & USA/asiático & $0,53 \pm 0,10$ & $7,92 \pm 3,14$ & $0,61 \pm 0,12$ & $11,67 \pm 2,81$ \\
\hline & 22 & USA/afroam, & $0,67 \pm 0,10$ & $8,94 \pm 2,3$ & $0,81 \pm 0,11$ & $13,06 \pm 3,18$ \\
\hline & 58 & USA/blanco & $0,52 \pm 0,12$ & $8,25 \pm 3,23$ & $0,64 \pm 0,12$ & $11,09 \pm 4,40$ \\
\hline Gomes $^{13}(2008)$ & 30 & Brasil & $0,81 \pm 0,31$ & - & $1,29 \pm 0,56$ & - \\
\hline Trindade $^{14}(2007)$ & 30 & Brasil & - & $8,78 \pm 2,12$ & - & $11,35 \pm 3,02$ \\
\hline $\operatorname{Dokic}^{15}(2010)$ & 100 & Macedonia & $0.71 \pm 0,07$ & $9,54 \pm 2,73$ & $0.87 \pm 1,12$ & $10.85 \pm 2.70$ \\
\hline Numminem ${ }^{16}(2002)$ & 249 & Finlandia & $0,57 \pm 0,18$ & $7,25 \pm 2,73$ & $0,69 \pm 0,10$ & $10,09 \pm 3,47$ \\
\hline Cankurtaran ${ }^{17}(2007)$ & 10 & Turquía & $0,48 \pm 0,16$ & - & $0,57 \pm 0,12$ & - \\
\hline Burres $^{18}(1999)$ & 28 & USA/asiático & $0,56 \pm 0,16$ & - & $0,67 \pm 0,12$ & - \\
\hline $\operatorname{Kim}^{19}(2007)$ & 153 & Korea & $0,42 \pm 0,19$ & - & $0,55 \pm 0,17$ & -- \\
\hline
\end{tabular}

nasal, mediante la medición de áreas mínimas de sección y volúmenes endonasales.

Los valores encontrados en este estudio mostraron diferencias estadísticamente significativas en los parámetros rinométricos al comparar sexo y respuesta posvasoconstricción, no encontrando en general diferencias importantes de éstos en la comparación con la antropometría.

Por el número de casos incluidos y la calidad de los datos, este estudio podría orientarse como un patrón de referencia nacional y respaldar estudios futuros con RA en patología rinosinusal.

\section{BIBLIOGRAFÍA}

1. Lee KJ, ed. Essential Otolaryngology, Head and Neck Surgery. $9^{\text {th }}$ ed. Mc Graw Hill, 2008.

2. Roithmann R, Cole P, Chapnik J, et al. Acoustic rhinometry in evaluation of nasal obstruction. Laryngoscope 1995; 105: 275-81.

3. Zeiders JW, Pallanch JF, Mc Caffery TV. Evaluation of nasal breathing function with objective airway testing. In: Cummings CW, Flint PW, Harker LA, Haughey BH, Richardson MA, Robbins RT, eds. Otolaryngology Head and Neck Surgery. $4^{\text {th }}$ ed. Baltimore: Elsevier; 2005; 898-930.

4. Clement PA, and GordTs F. Standardization committee on objective assessment of the nasal airway, IRS and ERS. Rhinology 2005; 43: 169-79.

5. Corey JP, Gungor A, Nelson R, Liu X, Fredberg J. Normative standards for nasal cross-sectional areas by race as measured by acoustic rhinometry. Otolaryngol Head Neck Surg 1998; 119(4): 389-93.

6. Pongsakorn Tantilipikorn, Perapun Jareoncharsri, Siriporn Voraprayoon, Chaweewan Bunnag, Peter A. Clement. Acoustic rhinometry of Asian noses. American Journal of Rhinology 2008; 22: 61720.

7. Miman MC, Deliktas H, Ozturan 0, Toplu Y, Akarçay M. Internal nasal valve: revisited with objective facts. Otolaryngol Head Neck Surg 2006; 134(1): 41.

8. Cakmak 0, Tarhan $\mathrm{E}$, Coskun M, Cankurtaran M, CELIK H. Acoustic rhinometry: accuracy and ability 
to detect changes in passage area at different locations in the nasal cavity. Ann Otol Rhinol Laryngol 2005; 114(12): 949-57.

9. Samolinski BK, Grzanka A, Gotlib T. Changes in nasal cavity dimensions in children and adults by gender and age. Laryngoscope 2007; 117(8): 1429-33.

10. MohebBi A, Farhadi M, Erfan A. Assessment of nasal volume and cross-sectional area by acoustic rhinometry in a sample of normal adult Iranians. Arch Iran Med 2008; 11(5): 555-8.

11. Tantilipikorn $P$, JareoncharsRi $P$, Voraprayoon $S$, Bunnag C, Clement PA. Acoustic rhinometry of Asian noses. Am J Rhinol 2008; 22(6): 617-20.

12. Straszek SP, Schlünssen V, Sigsgaard T, Pedersen OF. Reference values for acoustic rhinometry in decongested school children and adults: the most sensitive measurement for change in nasal patency. Rhinology 2007; 45(1): 36-9.

13. Gomes Ade 0, Sampaio-Teixeira AC, Trindade SH, TRINDADE IE. Nasal cavity geometry of healthy adults assessed using acoustic rhinometry. Braz J Otorhinolaryngol 2008; 74(5): 746-54.
14. Trindade IE, Gomes Ade 0, Sampaio-Teixeira AC, TRINDADE SH. Adult nasal volumes assessed by acoustic rhinometry. Braz J Otorhinolaryngol 2007; 73(1): 32-9.

15. Dokic $D$, Karkinski $D$, IsJanovska $R$, Trajkovska-Dokic E, FILIPCE I. Measuring nasal volumes with acoustic rhinometry. Prilozi 2010; 31(1): 339-47.

16. Numminen J, Ahtinen M $3^{\text {ro }}$, Huhtala $\mathrm{H}$, Laranne J, RAUTIAINEN M. Correlation between rhinometric measurement methods in healthy young adults. Am J Rhinol 2002; 16(4): 203-8.

17. Cankurtaran M, Celik H, Coskun M, Hizal E, Cakmak 0 . Acoustic rhinometry in healthy humans: accuracy of area estimates and ability to quantify certain anatomic structures in the nasal cavity. Ann Otol Rhinol Laryngol 2007; 116(12): 906-16.

18. BURRES SA. Acoustic rhinometry of the oriental nose. Am J Rhinol 1999; 13(5): 407-10.

19. KIm SW, Mo JH, KIm JW, KIm DY, Rhee CS, Lee $\mathrm{CH}$, MiN YG. Change of nasal function with aging in Korean. Acta Otolaryngol Supp/2007; (558): 90-4. 